\title{
Leishmaniasis cutánea inducida por especies de Leishmania Viannia en ratones BALB/c y eficacia de un tratamiento tópico
}

\section{Cutaneous leishmaniasis by Leishmania Viannia species in BALB/c mice and efficacy of a topical treatment}

\author{
Laura-F Neira $^{1}$; Diana-P Peña ${ }^{1}$; Angélica-M Vera ${ }^{1}$; Julio-C Mantilla ${ }^{1}$; Patricia Escobar ${ }^{1}$
}

Forma de citar: Neira LF, Peña DP, Vera AM, Mantilla JC, Escobar P. Leishmaniasis cutánea inducida por especies de Leishmania Viannia en ratones BALB/c y eficacia de un tratamiento tópico. Rev Univ Ind Santander Salud. 2019; 51(1): 33-42 doi: http://dx.doi.org/10.18273/revsal.v51n1-2019004 (c) (1)

\section{Resumen}

Introducción: La diversidad de las formas clínicas de la leishmaniasis del Nuevo Mundo (desde formas cutáneas localizadas a diseminadas o formas mucosas) causada por especies del subgénero Viannia podría inferir en la eficacia de los tratamientos tópicos. El objetivo del presente trabajo fue determinar las características de la leishmaniasis cutánea producida por infecciones con Leishmania (V.) braziliensis y L.(V.) panamensis en ratones BALB/c y la eficacia de un mismo tratamiento tópico. Materiales y métodos: Después de la infección con cada una de las especies se realizó seguimiento de las lesiones determinando su tamaño $\left(\mathrm{mm}^{2}\right)$ y características macroscópicas, cada siete días por 150 días. Las características histopatológicas (en lesiones y órganos) fueron determinadas 70, 106 y 150 días post-infección y la eficacia de un tratamiento tópico (cura de lesión y parasitológica) fue determinada después del tratamiento con un gel de miltefosina aplicado una vez al día por 20 días sobre las lesiones. Resultados: Se observó un aumento del tamaño de las lesiones en ambos grupos de ratones, sin embargo, un mayor tamaño de las lesiones e intensidad de la respuesta inflamatoria con menos alteraciones epidérmicas fue encontrada en los ratones infectados con $L$. (V.) braziliensis. En ningún grupo se encontraron parásitos en órganos (nódulos, bazo e hígado) ni diferencias en la efectividad del tratamiento tópico utilizado. Conclusión: La eficacia del tratamiento tópico utilizado no fue afectada por las diferencias macro y microscópicas encontradas en la leishmaniasis producida por las dos especies de Leishmania evaluadas.

Palabras clave: L.(V.) braziliensis; L.(V.) panamensis; Ratones BALB/c; Miltefosina; Patrones histopatológicos; Tratamiento tópico; Gel.

\begin{abstract}
Introduction: The efficacy of topical treatments could be affected by the diversity of clinical forms (localized or disseminated cutaneous forms, mucosal forms) of New World-leishmaniasis caused by species of Leishmania from the subgenus Viannia. The aim of this study was to determine the cutaneous leishmaniasis features produced after infection with Leishmania (V.) braziliensis and L. (V.) panamensis in BALB/c mice and to determine the efficacy of

1. Universidad Industrial de Santander, Bucaramanga, Colombia.

Correspondencia: Patricia Escobar. Dirección: $\mathrm{Km}^{2}$ Vía el Refugio sede UIS Guatiguará, Piedecuesta, Santander. Teléfono: +576340000. Correo electrónico: pescobar@uis.edu.co
\end{abstract}


one topical treatment. Materials and methods: Cutaneous leishmaniasis lesions were followed up after infection determining their lesion-size $\left(\mathrm{mm}^{2}\right)$ and other macroscopic characteristics every 7 days for 150 days. Histopathological patterns (in lesions and organs) were determined 70, 106 and 150 days post-infection and the efficacy (lesion and parasitological cure) of miltefosine gel applied topical once a day for 20 days was determined. Results: An increase of size-lesions was observed in both groups of mice, however, a higher lesion- size and inflammatory response but lower epidermal changes were observed in $L$. (V.) braziliensis compared with $L$. (V.) panamensis infected ones. No parasites were observed in organs (nodules, spleen and liver) and no differences were observed in the effectiveness of the used topical treatment. Conclusion: The efficacy of the topical treatment used was not affected by the macro and microscopic differences produced after infection by the two Leishmania species evaluated.

Keywords: L. (V.) braziliensis; L. (V.) panamensis; BALB/c mice; Miltefosine; Histopathological patterns; Topical treatment; Gel.

\section{Introducción}

Leishmaniasis son un conjunto de manifestaciones clínicas causadas por diferentes especies de protozoarios del género Leishmania y transmitida al hospedero mamífero a través de insectos flebótomos. Sus formas clínicas dependen de la especie de parásito involucrada y del estado inmune del hospedero siendo la Leishmaniasis Cutánea (LC) el síndrome más común con una prevalencia de 0,7-1,2 millones de casos a nivel mundial ${ }^{1}$. El tratamiento se basa en el uso parenteral de antimoniales pentavalentes $\left(\mathrm{Sb}^{\mathrm{v}}\right)$ y en caso de falla terapéutica o contraindicaciones se utiliza la anfotericina B (AmB) y sus formas lipídicas, el isetionato de pentamidina, el sulfato de paromomicina y la miltefosina. Estos tratamientos convencionales sistémicos presentan eficacia variable, efectos tóxicos como pancreatitis, falla cardiaca y renal y están contraindicados en mujeres embarazadas e inmunosuprimidos. Generalmente son aplicados en protocolos largos y dolorosos, en puestos de salud alejados a las viviendas lo cual hace que los pacientes abandonen el tratamiento (no adherencia) ocasionando falla teraputica ${ }^{2}$. Los tratamientos tópicos constituyen una alternativa atractiva, de fácil administración, bajo costo y toxicidad, de gran utilidad en pacientes con lesiones tempranas, pequeñas y únicas ${ }^{3-4}$. No obstante, deben ser aplicados con precaución en pacientes con leishmaniasis causadas por especies dermotrópicas del Nuevo Mundo (especies de Leishmania del subgénero Viannia) debido al potencial riesgo de diseminación y/o la heterogeneidad clínica ${ }^{4}$. Algunos tratamientos tópicos como termoterapia, electroterapia o la terapia fotodinámica y el uso de formulaciones tipo ungüentos o cremas conteniendo paromomicina, imiquimod o $\mathrm{AmB}$ han mostrado efectividad en algunos casos de $\mathrm{LC}^{3-4}$. Sin embargo, no hay un tratamiento ideal en LC, pues el fármaco, la dosis y el tiempo de tratamiento debe ser individualizado dependiendo de la especie de parásito involucrada, de las características del huésped y las características clínicas de la enfermedad 5 .
La leishmaniasis del Nuevo Mundo (leishmaniasis tegumentaria americana) es multiforme en sus manifestaciones clínicas (de lesiones cutáneas localizadas a formas diseminadas y/o formas destructivas de las mucosas) ${ }^{5-7}$. Las lesiones cutáneas generalmente son indoloras y crónicas auto-limitadas afectando principalmente zonas expuestas a los vectores como la cara y extremidades. Pueden resolverse espontáneamente o formar lesiones no cicatrizantes que requieren tratamiento. Inician como una pápula eritematosa que evoluciona a un nódulo que se ulcera pudiendo ser únicas o múltiples en varias áreas del cuerpo resultantes de la diseminación hematógena o linfática del parásito ${ }^{7}$. Microscópicamente se observan diferentes patrones histológicos en humanos siendo el más común el de dermatitis difusa ( $85 \%$ de los pacientes con LC) que muestra una epidermis hiperplásica, ulcerada, escamocostras e infiltrado dérmico difuso con macrófagos vacuolados, plasmocitos, linfocitos y amastigotes intracelulares en las papilas dérmicas. Otros patrones como el de dermatitis granulomatosa (de granulomas epitelioides, linfocitos y plasmocitos y escasas células gigantes y amastigotes), el de dermatitis granulomatosa tipo tuberculoide (con vasculitis y áreas de necrosis fibrinoide) o el de dermatitis difusa con epidermis normal o atrófica y macrófagos vacuolados conteniendo abundante amastigotes, pueden ser observados $^{7-8}$.

La infección por $L$. (V.) panamensis en humanos se presenta inicialmente como una pápula que después de 1-2 semanas empieza a evolucionar hasta llegar a una lesión ulcerada de bordes elevados algunas veces con pequeñas lesiones satélites y adenopatía localizada. La lesión incrementa lentamente de tamaño y profundidad y se auto-limita (la mayoría de las veces) después de cuatro meses de seguimiento ${ }^{7,9}$. Los amastigotes se encuentran intracelularmente en los macrófagos de la región superior de la dermis (adyacente a la epidermis) observándose áreas con necrosis. El número de 
amastigotes es mayor en la lesión papular inicial que en la úlcera. El perfil inflamatorio revela la predominancia de macrófagos, linfocitos en la unión epidermis-dermis, algunos granulomas y áreas con necrosis. También se observan células plasmáticas, células gigantes y vasculitis $^{6-8}$. La LC producida por $L$. (V.) braziliensis ha mostrado lesiones nodulares eritematosas, induradas con ulceración central y presencia de exudado ${ }^{6}$. Microscópicamente se observa infiltrado dérmico inflamatorio mixto superficial con presencia de linfocitos, células plasmáticas, histiocitos y células epiteliodes con epidermis ulcerada superpuesta, encontrándose macrófagos parasitados con amastigotes $^{6-7}$. Las diferencias clínicas e histopatológicas entre la infección con L.(V.) panamensis vs L.(V.) braziliensis en el modelo murino, podrían estar involucradas en la respuesta a los tratamientos tópicos. Este trabajo pretende determinar estas características de la LC producida por infecciones con estas dos especies de Leishmania del genero Viannia en ratones BALB/c y la eficacia de una misma formulación tópica.

\section{Materiales y métodos}

Parásitos: Se utilizaron promastigotes de $L$. (V.) braziliensis (MHOM/BR/75/M2903) y $L . \quad(V$.) panamensis (MHOM/PA/71/LS94) los cuales fueron mantenidos en medio Drosophila de Schneider (Sigma Aldrich ${ }^{\circledR}$, St. Louis, USA) suplementado con $10 \%$ de suero bovino fetal inactivado con calor (SBFi, Gibco, Grand Island, USA), a $26^{\circ} \mathrm{C}$.

Ratones, infección y sacrificio: Se utilizaron ratones BALB/c de 8-10 semanas los cuales fueron mantenidos en condiciones controladas de temperatura, humedad, comida y agua ad libitum. Los estudios fueron desarrollados de acuerdo a la guía y protocolos para el cuidado y uso de animales de laboratorio aprobado por el Comité de Ética (Código D16-00730) de la Universidad Industrial de Santander.

Los ratones $\mathrm{BALB} / \mathrm{c}$ fueron infectados con $5 \times 10^{5}$ promastigotes/ratón en el dorso, vía subcutánea. Un grupo $(\mathrm{N}=7)$ fue infectado con $L .(V$.$) panamensis y otro$ $(\mathrm{N}=7)$ con L. (V.) braziliensis.

Los animales fueron sacrificados utilizando una mezcla de anestésicos (Xilacina y Ketamina) a los $70(\mathrm{~N}=2), 106(\mathrm{~N}=2)$ y $150(\mathrm{~N}=3)$ días post infección (p.i). Se realizaron improntas para determinar la presencia del parásito y se recolectaron fragmentos de las lesiones, piel, bazo, hígado y ganglios linfáticos los cuales fueron colocados en una solución de formalina tamponada al $10 \%(\mathrm{pH} 7,0)$ para estudios histopatológicos. Las improntas fueron coloreadas con Giemsa y las biopsias fueron deshidratadas e incluidas en bloques de parafina para ser cortadas con micrótomo $(5 \mu \mathrm{m})$ y posteriormente coloreadas con hematoxilina y eosina.

Evolución macroscópica de las lesiones postinfección (p.i.): Fue determinada, ratones infectados con L. (V.) panamensis $(\mathrm{N}=7)$ y con $L$. (V.) braziliensis $(\mathrm{N}=7)$. A partir de su aparición, las lesiones (nódulos y/o úlceras) empezaron a medirse utilizando un caliper digital cada siete días hasta el fin del experimento (150 días p.i.). El área de las lesiones $\left(\mathrm{mm}^{2}\right)$ fue calculada y se realizó registro fotográfico. Se determinó el delta o velocidad de crecimiento de las lesiones mediante el cociente de la resta de los valores de tamaño y tiempo de evolución de las lesiones $\left(\mathrm{mm}^{2} / \mathrm{día}\right)$.

Evaluación histopatológica de las lesiones: Fue determinada 70, 106 y 150 días p.i. En la epidermis se determinó la presencia de acantosis (aumento en la proliferación celular), hiperqueratosis (engrosamiento del estrato corneo y aumento de queratina), paraqueratosis (núcleos en las células del estrato corneo) y espongiosis (visualización de los desmosomas). En la dermis se determinó la presencia de infiltrado inflamatorio (invasión de leucocitos), de granulomas (acúmulo de células inflamatorias), de fibrina y leucocitos y la presencia de células plasmáticas, macrófagos, linfocitos, eosinófilos, polimorfonucleares y bacterias. En el bazo se determinó el estado de los nódulos esplénicos, el infiltrado celular y la presencia de células plasmáticas. En el hígado la presencia de granulomas, fibrosis y aglomeración de sinusoides. En los ganglios poplíteos se determinó la respuesta inflamatoria. En todos los órganos se determinó la presencia de amastigotes.

Los resultados se expresaron utilizando una escala semi-cuantitativa ajustada a cada patrón histopatológico teniendo en cuenta tanto el grado de la variable leve (1-15/campo), moderado (16-30/campo) o severo (>30/campo) en 50 campos microscópicos ${ }^{17}$.

Determinación de la eficacia del tratamiento tópico: Dos grupos de ratones con LC de cada especie $(\mathrm{N}=2)$ fueron tratados tópicamente a los 70 días p.i con una formulación tipo gel conteniendo $0,5 \%$ de miltefosina (MTF) (Cayman, Michigan, USA) aplicando una dosis día por 20 días $^{20}$. El tamaño de las lesiones fue determinado semanalmente y la efectividad del tratamiento fue determinada dividiendo el tamaño 
de las lesiones antes del tratamiento con el tamaño 15 días después del fin del tratamiento x 100. Los resultados fueron comparados con el grupo control. La presencia de parásitos se evaluó microscópicamente en las improntas de las lesiones y órganos coloreadas con Giemsa.

Análisis de resultados: Los resultados fueron analizados utilizando el software GraphPad Prism $\mathbb{R}$ Version 6,0 (GraphPad Software Inc. San Diego,CA, USA). Los datos fueron expresados con la media \pm desviación estándar (DS). La evolución del tamaño de lesiones de cada grupo fue analizada utilizando la prueba $\mathrm{t}$ de Student múltiple. Valores de $\mathrm{p}<0,05$ fueron considerados estadísticamente significativos. Los resultados del análisis histopatológico fueron analizados por dos observadores independientes obteniendo el índice de concordancia Kappa para las variables estudiadas.

\section{Resultados}

Evolución de las lesiones: La evolución de las lesiones (nódulo y úlcera) en las dos especies de Leishmania se muestran en la Figura 1. Se registró un aumento progresivo del tamaño de las lesiones en los diferentes días hasta los 150 días de observación. Se encontraron diferencias estadísticamente significativas $(p<0,05)$ en el tamaño de las lesiones entre las dos especies de Leishmania siendo de mayor tamaño las producidas por L. (V.) braziliensis que las de $L$. (V.) panamensis. En los días 70, 106 y 150 p.i. el tamaño promedio de los nódulos en infecciones por $L$. (V) braziliensis fue de 44,$1 ; 67,4 ; 106,7 \mathrm{~mm}^{2}$ en y de 25,$2 ; 55,2 ; 93,6 \mathrm{~mm}^{2}$ en los infectados con $L$. (V.) panamensis. El tamaño promedio de las úlceras fue de 4,$2 ; 10,5 ; 24,1 \mathrm{~mm}^{2}$ en $L$. ( $V$.) braziliensis y 2,$1 ; 5,9 ; 10,13 \mathrm{~mm}^{2}$ en $L$. (V.) panamensis.

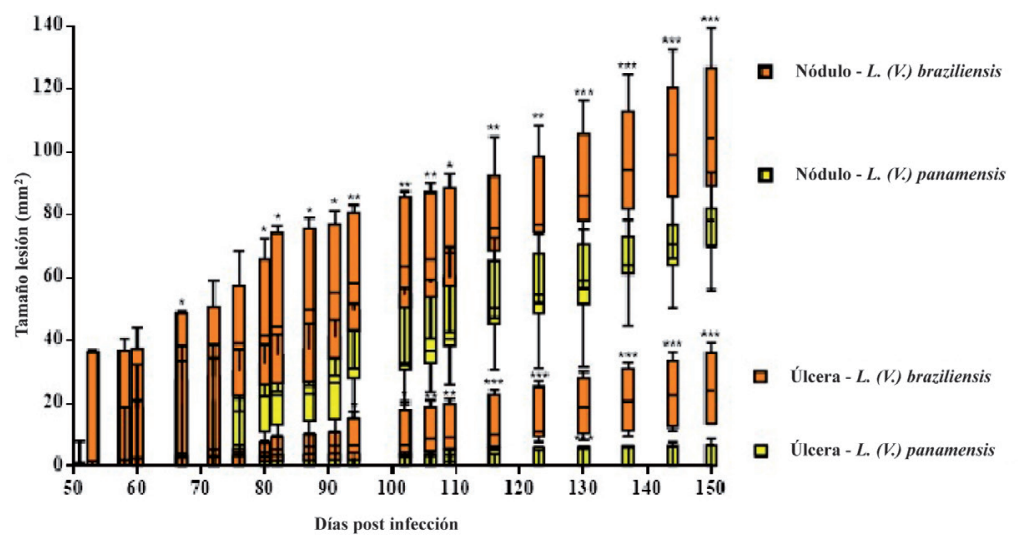

Figura 1. Evolución de las lesiones producidas en ratones BALB/c infectados con Leishmania. La figura muestra en diferentes días post-infección el área $(\mathrm{mm} 2)$ del nódulo y de la úlcera que presentaron los ratones infectados con $L$. (V.) panamensis (amarillo) y L. (V.) braziliensis (naranja) *Diferencias significativas $\mathrm{p}<0,05 * * \mathrm{p}<0,01 * * * \mathrm{p}<0,005$.

Características macroscópicas de las lesiones: Las lesiones de los ratones infectados con L. (V.) panamensis iniciaron macroscópicamente como una pápula 62-67 días p.i. la cual se transformó en un nódulo elevado e indurado de morfología irregular. La formación de úlcera fue evidenciada en el $57 \%$ de los ratones infectados presentándose como una leve a moderada invaginación recubierta de piel con centro eritematoso y con formación de costra en algunas de ellas (Figura 2A). La velocidad de crecimiento promedio del nódulo fue $0,3 \mathrm{~mm}^{2} /$ día.

Las lesiones en los ratones infectados con L.(V.) braziliensis empezaron a evidenciarse 50-60 días p.i. (primero que en las infecciones por $L$. $(V$.) panamensis) con la formación de una pápula que se transformó en un nódulo regular (ovoide) y menor elevación que en $L$. $(V$.) panamensis. El 100\% de los ratones formó una úlcera central, de bordes eritematosos y fondo necrótico. La velocidad de crecimiento promedio del nódulo fue de $0,45 \mathrm{~mm}^{2} /$ día. Algunas lesiones presentaron secreción purulenta y eritema en su periferia. La formación de costra se observó en todos los ratones infectados (Figura 2B).

En todas las improntas de las lesiones se observaron microscópicamente la presencia de abundantes amastigotes extra e intracelulares (Figura 2). En las improntas de órganos no se observaron parásitos.

Análisis histopatológico: Después de la infección y dependiendo de los días en que fueron sacrificados los animales (70, 106 y 150 días p.i.), se observaron cambios estructurales en las diferentes capas de la piel 
(epidermis, dermis e hipodermis) como se muestra en la Figura 3 para $L$. (V.) panamensis y Figura 4 en $L$. (V.) braziliensis. En la Figura $\mathbf{3 A}$ y $4 \mathbf{A}$ se muestra la estructura de piel normal distinguiéndose la capa basal, granulosa y el estrato corneo de la epidermis, el tejido conjuntivo laxo irregular con fibras de colágeno, fibroblastos y macrófagos dispersos de la dermis; el tejido graso de la hipodermis y el tejido muscular adyacente (Figura 3A y 4A).
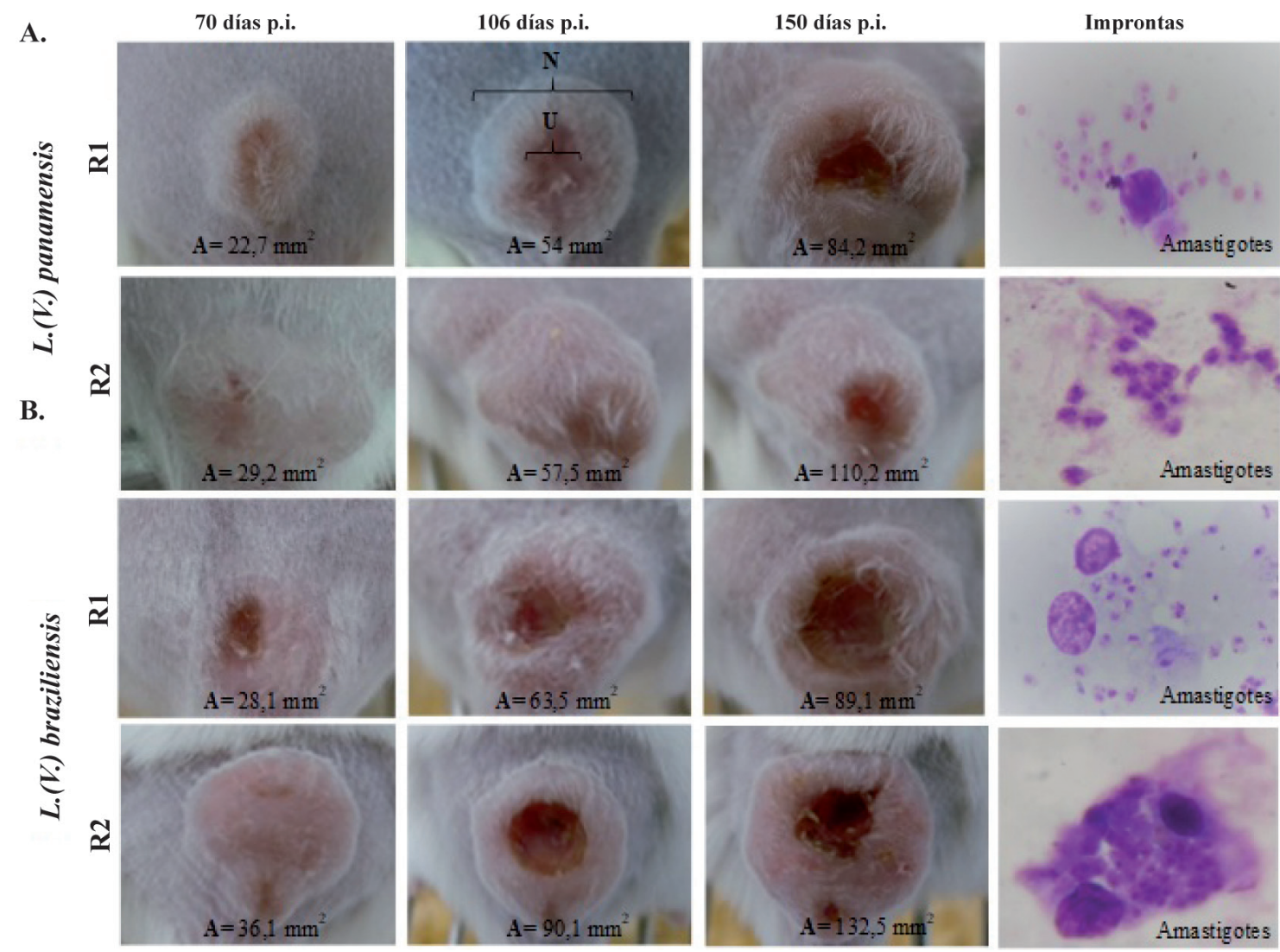

Figura 2. Lesiones producidas en ratones BALB/c infectados con Leishmania. Fotografía de las lesiones cutáneas de ratones (R1 y R2) infectados con L. (V.) panamensis (A.) y de ratones (R1 y R2) infectados con L. (V.) braziliensis (B.), 70,106 y 150 días post-infección. En la última columna, se muestran microfotografías (aumento 1000X) de improntas de las lesiones con amastigotes. A: área del nódulo en mm2. N:nódulo, U:úlcera

En las infecciones con L.(V.) panamensis, 70 días p.i., se observó una leve acantosis o aumento de las capas de la epidermis; la dermis se observó definida con leve infiltrado inflamatorio difuso predominante de linfocitos, polimorfonucleares, cantidad moderada de macrófagos infectados $\mathrm{y}$ amastigotes extracelulares visualizados hasta el tejido muscular (Figura 3B y 3C). No se observaron focos evidentes de necrosis, ni sobreinfección bacteriana, ni cambios en los folículos pilosos y las glándulas sebáceas. A los 106 días p.i. se evidenció moderada hiperqueratosis y acantosis en la epidermis, aumento del estrato espinoso y leve espongiosis (separaciones en las uniones intracelulares). La dermis y la hipodermis perdieron su delimitación y se visualizó un severo infiltrado inflamatorio difuso con predominio de linfocitos, células plasmáticas y abundantes macrófagos infectados con amastigotes (Figura 3D y 3E). A los 150 días p.i. las alteraciones en dermis fueron similares, sin embargo, las alteraciones epidérmicas fueron más visibles con severa acantosis y espongiosis, moderada hiperqueratosis y pérdida de la unión dermoepidérmica (Figura 3F).

En ratones infectados con $L$. (V.) braziliensis, se observó leve acantosis e hiperqueratosis en la epidermis a los 70 p.i. moderado infiltrado inflamatorio difuso con predominio de polimorfonucleares dispuesto en focos de inflamación rodeados de una cantidad moderada de macrófagos infectados y amastigotes extracelulares en la dermis y pérdida de la delimitación entre dermis e hipodermis (Figura 4B a C). A los 106 y 150 días p.i. el patrón histopatológico fue similar. En epidermis se 
observó moderada paraqueratosis e hiperqueratosis sin acantosis, en dermis el infiltrado inflamatorio difuso fue severo con predominio de linfocitos y células plasmáticas, algunos polimorfonucleares y focos de necrosis (Figura 4D a F), se observaron abundantes macrófagos infectados y amastigotes extracelulares. En la Tabla 1, se muestra la intensidad de cada una de las variables.
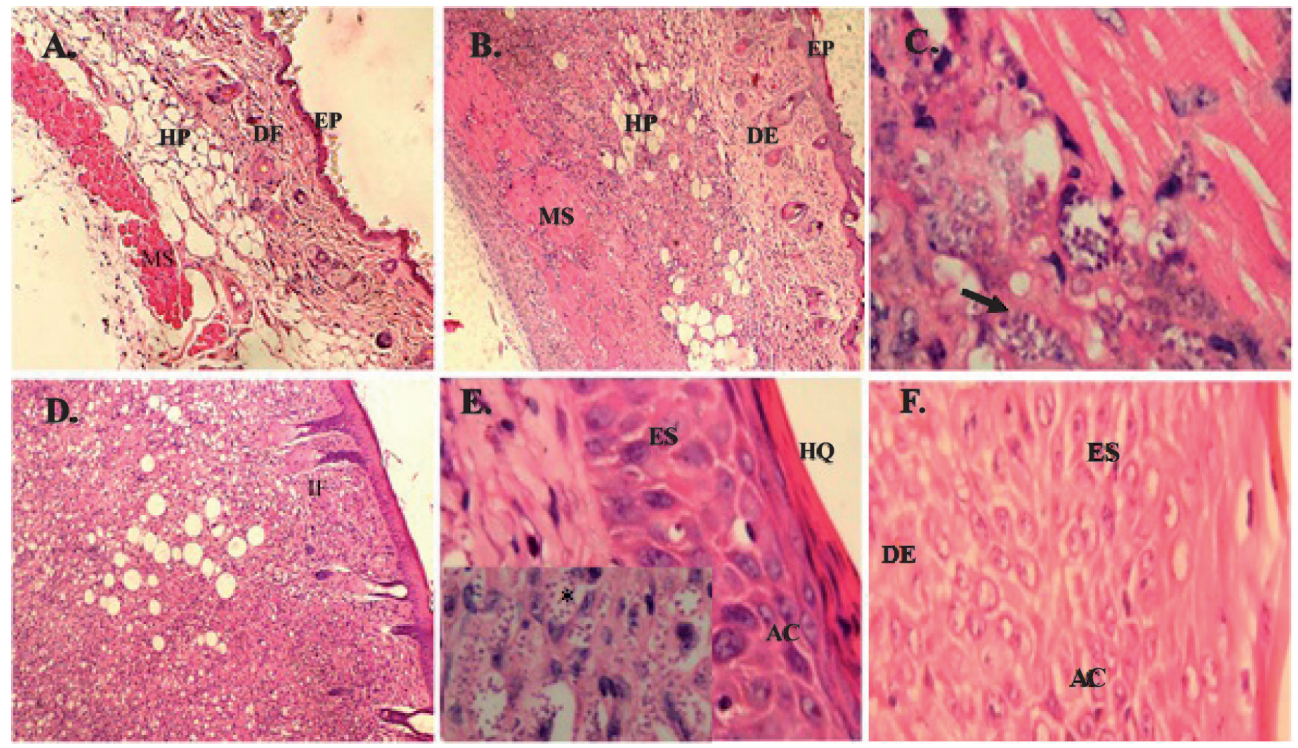

Figura 3. Características histopatológicas de lesiones producidas por L. (V.) panamensis. A. Piel normal; B. 70 días p.i. infiltrado inflamatorio en la hipodermis, aumento 100X; C. La flecha indica macrófagos infectados, 1000X; D. 106 días p.i, infiltrado inflamatorio difuso con abundantes amastigotes (recuadro), 400X; E. 150 días p.i Alteraciones en epidermis 1000X. F. Perdida unión dermo-epidérmica, 1000X. EP: epidermis, DE: dermis, HP: hipodermis, MS: músculo, IF: infiltrado inflamatorio, ES: espongiosis, HQ: hiperqueratosis, AC: acantosis, (*): amastigotes. Las biopsias fueron analizadas por dos observadores, $\mathrm{K}$ de 0,6 con un grado de acuerdo sustancial
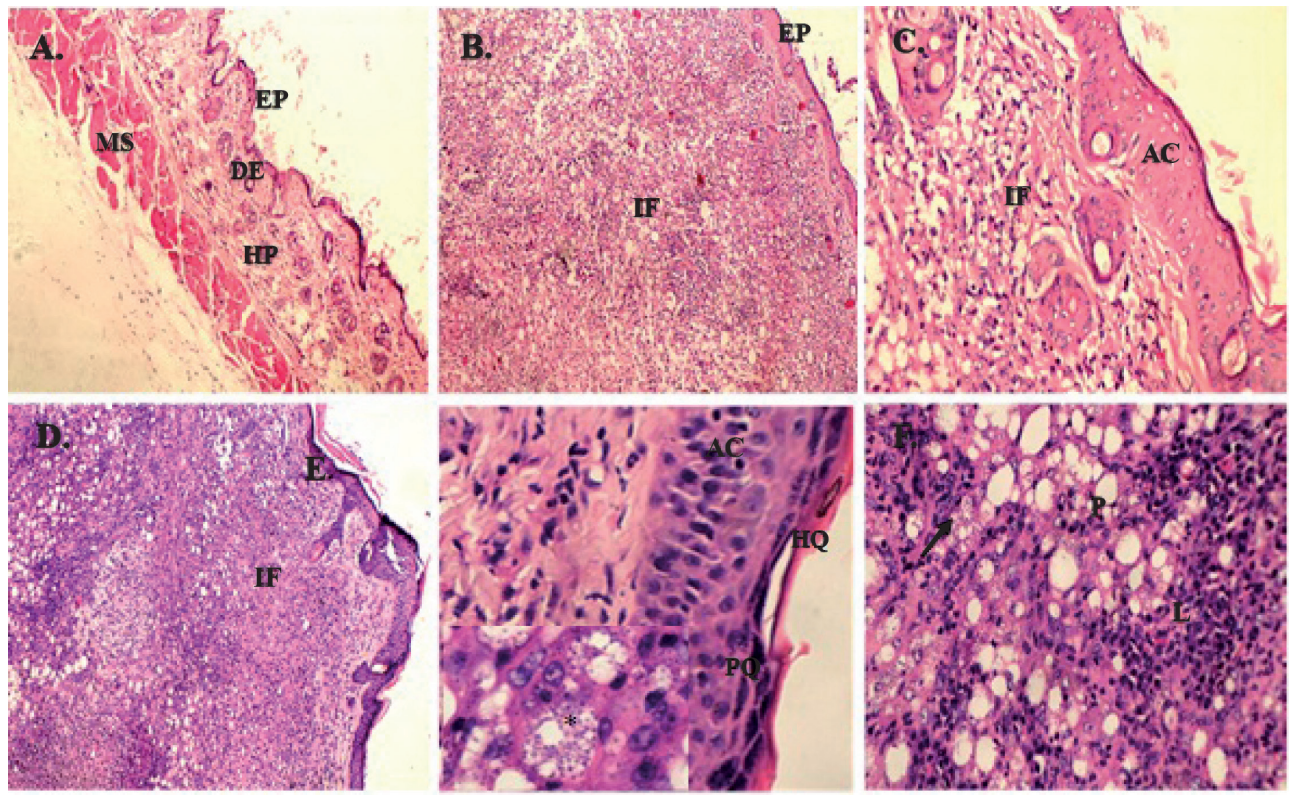

Figura 4. Características histopatológicas de lesiones producidas por L. $(V$.) braziliensis. A. Piel normal; B. 70 días p.i. infiltrado inflamatorio difuso, aumento 100X; C. 106 p.i., proliferación celular en epidermis e infiltrado inflamatorio 400X; D. 150 días p.i. infiltrado inflamatorio difuso; E. Alteración en la epidermis, acantosis, aumento 1000X; F. La flecha indica un macrófago infectado, infiltrado inflamatorio de linfocitos basófilos y plasmocitos, aumento 400X. EP: epidermis, DE: dermis, HP: hipodermis, MS: musculo, IF: infiltrado inflamatorio, PQ: paraqueratosis, HQ: hiperqueratosis, AC: acantosis, L: linfocitos, $\mathrm{P}$ : células plasmáticas. $\mathrm{K}$ de 0,7 con un grado de acuerdo sustancial. 
En los órganos estudiados hígado, bazo y ganglio se observaron cambios histopatológicos similares para ambas especies. En el hígado se observó ligera aglomeración de sinusoides, linfocitos y polimorfonucleares, sin fibrosis o granulomas, en el bazo se observó una ligera pérdida de la proporción entre la pulpa roja y la pulpa blanca con aumento de folículos esplénicos, linfocitos y en la pulpa roja con aumento de eritrocitos, megacariocitos y leucocitos. Los ganglios se observaron aumentados de tamaño y microscópicamente se observó un aumento linfocitos acompañados de células dendríticas; no se observó la medula ni el hilio del ganglio. En ningún órgano se observaron amastigotes (intracelulares o extracelulares) en los campos microscópicos observados (datos no mostrados).

Tabla 1. Intensidad de las variables histopatológicas estudiadas en lesiones de ratones infectados con $L$. ( $V$.) panamensis y $L$. ( V.) braziliensis. La tabla muestra la intensidad [+(1-15/campo):leve, $++(16-30 /$ campo): moderado, $+++(>30 /$ campo):severo] de las variables encontradas en E: epidermis y D: dermis, 70, 106 y 150 días post-infección. Los cuadros amarillos para $L$. (V.) panamensis y los anaranjados para $L$. (V.) braziliensis muestran las características más sobresalientes. (-): ausencia de la variable en los campos observados. R: ratón. Observación en 50 campos microscópicos, aumentos de 1000x.

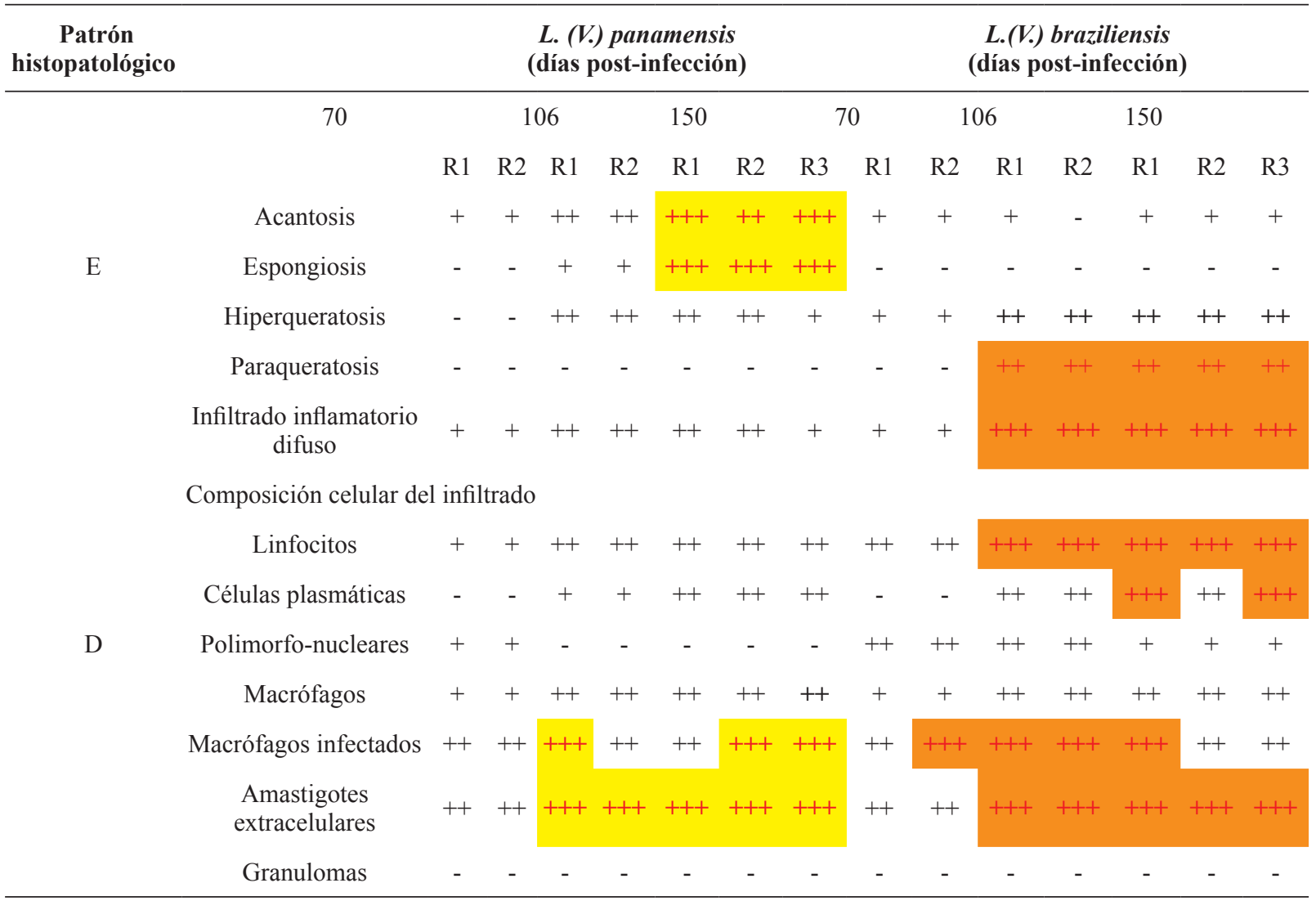

Eficacia del tratamiento tópico: El tratamiento tópico con el gel de MTF indujo una disminución en el tamaño de las lesiones producidas por $L$. (V.) panamensis y L. (V.) braziliensis de $23,3 \pm 0.5$ a $0 \mathrm{~mm}^{2}(100 \%$ de disminución) y de $22,6 \pm 0,3$ a $1,6 \pm 0,1 \mathrm{~mm}^{2}(91 \%$ disminución) respectivamente, 15 días después del fin del tratamiento (resultado no mostrado). Por el contrario, en el grupo control no tratado se observó un aumento de las lesiones de 22,9 $\pm 0,8$ a 47,5 $\pm 1,1 \mathrm{~mm}^{2}$ en $L$.(V.) panamensis y de 21,6 $\pm 1,1$ a $49,4 \pm 1,5 \mathrm{~mm}^{2}$ en $L$. $(V$.) braziliensis. No se observaron parásitos en las improntas de lesión y órganos (bazo, hígado y ganglio) de los animales tratados con la formulación de MTF. En los ratones infectados con L.(V.) panamensis después del tratamiento se recuperó la epidermis (ausencia de acantosis, espongiosis y paraqueratosis); en la dermis se observó leve infiltrado inflamatorio difuso con predominio de linfocitos y células plasmáticas con ausencia de amastigotes en todos los campos evaluados. En los ratones infectados con L.(V.) braziliensis, se observó una ligera hiperqueratosis; en la dermis se observó infiltrado inflamatorio difuso moderado mayor en comparación con los ratones infectados con $L$. $(V$.) panamensis después del tratamiento. 


\section{Discusion}

Los modelos murinos en LC utilizando las especies de parásitos que afectan a los humanos, nos permiten entender la enfermedad y evaluar la efectividad de nuevos fármacos. Esto a diferencia de lo que ocurre por ejemplo en el modelo experimental murino en malaria (especificidad del parásito por el huésped), o inclusive en el modelo experimental en Trypanosoma cruzi en donde es difícil lograr una infección crónica en los ratones ${ }^{10}$. En este trabajo se implementaron dos modelos experimentales producidos por especies involucradas en LC del Nuevo Mundo. Los dos modelos, presentaron similitudes y algunas diferencias importantes entre sí. Se encontró por ejemplo que el tamaño de las lesiones fue mayor en las infecciones por $L$. (V.) braziliensis, que las alteraciones epidérmicas (acantosis y espongiosis) fueron más evidentes en infecciones con $L$. (V.) panamensis y que la respuesta inflamatoria fue mayor en los animales infectados con L.(V.) braziliensis. Sin embargo, la intensidad de la infección (macrófagos infectados y amastigotes extracelulares) y la hiperqueratosis en la epidermis entre otros fue similar. En general, como algo muy positivo, los modelos establecidos mostraron similitudes a lo encontrado en pacientes en lo referente a la fase silenciosa o asintomática (sin lesión o inflamación aparente) la cual fue de aproximadamente de 50 días y a la fase de desarrollo de las lesiones (lesiones nodulares y ulcerativas muy similares a las de humanos) histológicamente con migración y activación de macrófagos, polimorfonucleares y migración de células dendríticas y linfocitos) ${ }^{11}$. Sin embargo, la fase crónica caracterizada en humanos por la resolución de la lesión asociada con la persistencia del parásito en los macrófagos y la inmunidad, no se logró totalmente en el modelo, al menos en el tiempo máximo de estudio (150 días p.i.), aunque, los aspectos relacionados con la respuesta inmune no fueron estudiados. La fase crónica de $\mathrm{LC}$ en ratones $\mathrm{BALB} / \mathrm{c}$ fue demostrada en un estudio por otros autores utilizando algunas condiciones diferentes tales como la infección con un número bajo de promastigotes metacíclicos $\left(5 \times 10^{4}\right)$ y un aislado diferente de $L$. (V.) panamensis ${ }^{12}$, observaron un inicio de lesiones relativamente rápido (20 días p.i.) que incrementaron hasta los 60 días p.i. con cronicidad (auto-limitadas) por 350 días, parásitos en lesión, nódulos linfáticos y en algunos en órganos (bazo, hígado), sin signos de cura espontanea ${ }^{12}$. Las lesiones en nuestro modelo no fueron auto-limitadas (al menos en el tiempo evaluado continuaban creciendo) y no se observaron parásitos en ganglios, bazo e hígado en los campos microscópicos observados. En LC experimental, factores como el número de parásitos, sitio de infección (oreja, patas, lomo, hocico), especie y fase del ciclo del parásito (amastigotes, promastigotes metacíclicos o no) o sexo, genética, edad, peso de los ratones podrían estar afectando la evolución de la LC, dificultando también la comparación de resultados ${ }^{11}$. Por ejemplo, algunos autores han demostrado que infecciones con $L$. $\left(V\right.$.) panamensis $\left(10^{7}-10^{8}\right.$ parásitos en la almohadilla plantar) desarrollaron lesiones de apariencia tumoral sin ulceración, necrosis o metástasis 160 días p.i. presentando un infiltrado inflamatorio extenso con macrófagos vacuolados (similar a tejido adiposo) conteniendo abundantes parásitos y necrosis focalizada, sin cambios aparentes en la epidermis (sin acantosis e hiperplasia), sin parásitos en hígado y bazo pero con parásitos en nódulos linfáticos ${ }^{13}$. Además, algunos autores han evidenciado lesiones secundarias en dedos, talones, cola y orejas en hámsteres (Mesocricetus auratus) tres meses p.i. relacionado con la cepa de $L$. (V.) panamensis y la baja producción de citoquinas como interferón (IFN)- $\gamma^{14}$.

Las infecciones con $L$. (V.) braziliensis en ratones BALB/c han mostrado resultados diversos (diferencias en la evolución de las lesiones inclusive en infecciones con aislados de la misma especie), generalmente sin producción de lesiones graves (lesiones nodulares, no ulceradas) no duraderas (curación espontánea), algunas con permanencia de parásitos en los nódulos linfáticos o con diseminación de estos a otros órganos ${ }^{15-17}$. En un estudio realizado en ratones infectados con promastigotes en fase estacionaria $\left(10^{5}\right.$ en la oreja) de $L$. (V.) braziliensis mostraron lesiones nodulares y ulceradas a partir de los 21 días p.i. las cuales aumentaron hasta el día 35 p.i. sin embargo, a diferencia del modelo nuestro, estas curaron espontáneamente y cicatrizaron 63 días p.i. con presencia de parásitos en lesiones y también en nódulos linfaticos ${ }^{16}$. Es interesante que los parásitos permanecieron en nódulos linfáticos (más no en las lesiones) hasta el final del experimento (70 días p.i.), adicional a esto la respuesta inflamatoria fue similar a la nuestra, iniciando con polimorfonucleares, macrófagos infectados parasitados seguido de cambios vasculares, necrosis tisular con posterior presencia de macrófagos, células epitelioides, linfocitos, células plasmáticas y parásitos; sin embargo a los 63 días p.i. (concomitante con el proceso de cicatrización) se observó depósito de fibras de colágeno ${ }^{16}$. Esta resolución de las lesiones también fue demostrada en un estudio en ratones infectados con $10^{7}$ promastigotes en la almohadilla plantar presentando lesiones moderadas (nodulares y no-ulceradas) con aumento inicial del número de parásitos y del infiltrado inflamatorio (con 
frecuencia de pequeños granulomas nodulares) pero con disminución progresiva de lesiones y parásitos, del infiltrado inflamatorio (macrófagos epiteloides, eosinófilos, linfocitos y células plasmáticas), de los amastigotes intracelulares pero evidenciando una fibroplasia (fibrosis, cicatrización), 70 días p.i. ${ }^{17}$. Las razones del porque las LC en nuestro modelo fueron diferentes a los dos casos anteriores están en estudio. Es posible que la respuesta inmune de los ratones o algunos factores de virulencia adquiridos por los parásitos estén involucrados.

Las diferencias encontradas en las lesiones entre las dos especies estudiadas, no afectaron estadísticamente la efectividad del tratamiento tópico con el gel de miltefosina (aplicado en iguales condiciones, igual dosis, tiempo de tratamiento, hora de aplicación). Diferentes tasas de curación que van del $95 \%$ en leishmaniasis visceral causada por $L$. donovani, del $53 \%$ en pacientes infectados con L.(V.) braziliensis y de $91 \%$ para $L .(V$.) panamensis en las regiones de Panamá, Guatemala y Colombia han sido reportadas ${ }^{19-20}$. La efectividad del tratamiento tópico de la miltefosina ha mostrado ser variable, dependiendo de las características del sistema farmacéutico utilizado y de la especie de Leishmania ${ }^{21}$. La idea que los tratamientos tópicos puedan brindar efectividad en casos de LC con diferentes grados de la enfermedad sería un gran alivio para los pacientes, sin embargo, estos deberán utilizarse preferiblemente en combinación con un tratamiento sistémico (seguramente este en dosis menos tóxicas) para evitar la inducción de resistencia y el riesgo de complicaciones.

\section{Conclusiones}

Se encontraron algunas diferencias en la evolución y en las características macro e histopatológicas entre las lesiones producidas por $L$. (V.) panamensis y $L$. $(V$.$) braziliensis en ratones BALB/c. La eficacia de un$ tratamiento tópico (gel de miltefosina) no fue afectada por estas diferencias.

\section{Agradecimientos}

Este proyecto fue financiado con recursos del Patrimonio Autónomo Fondo Nacional de Financiamiento para la Ciencia la Tecnología y la Innovación Francisco José de Caldas, Contrato RC-687-2014 y de la Universidad Industrial de Santander. Agradecemos a Jorge Armando Osorio por el apoyo en el mantenimiento y cuidado de los animales y a los miembros del laboratorio de Patología de la Facultad de Salud de la Universidad Industrial de Santander por el apoyo en el procesamiento de las biopsias.

\section{Conflicto de interés}

Los autores declaran que no hay conflicto de interés en el trabajo realizado

\section{Referencias}

1. Alvar J, Vélez ID, Bern C, Herrero M, Desjeux P, Cano J, et al. Leishmaniasis worldwide and global estimates of its incidence. PLoS One. 2012; 7(5): e35671. doi: 10.1371/journal.pone.0035671.

2. Sundar S, Chakravarty J. Antimony toxicity. Int J Environ Res Public Health. 2010; 7(12): 42674277. doi: 10.3390/ijerph7124267.

3. Wolf-Nassif P, de Mello TFP, Navasconi TR, Mota CA, Demarchi IG, Aristides SMA, et al. Safety and efficacy of current alternatives in the topical treatment of cutaneous leishmaniasis: a systematic review. Parasitology. 2017; 144(8): 995-1004. doi: 10.1017/S0031182017000385.

4. Blum J, Lockwood DN, Visser L, Harms G, Bailey MS, Caumes E, et al. Local or systemic treatment for New World cutaneous leishmaniasis? Re-evaluating the evidence for the risk of mucosal leishmaniasis. Int Health. 2012; 4(3): 153-163.

5. Aronson N, Herwaldt BL, Libman M, Pearson R, Lopez-Velez R, Weina P, et al. Diagnosis and treatment of leishmaniasis: Clinical Practice Guidelines by the Infectious Diseases Society of America (IDSA) and the American Society of Tropical Medicine and Hygiene (ASTMH). Am J Trop Med Hyg. 2017; 96(1): 24-45. doi: 10.4269/ ajtmh.16-84256.

6. Silva J, Queiroz A, Moura I, Sousa RS, Guimarães LH, Machado PRL, et al. Dynamics of American tegumentary leishmaniasis in a highly endemic region for Leishmania (Viannia) braziliensis infection in northeast Brazil. PLoS Negl Trop Dis. 2017; 11(11): e0006015. doi: 10.1371/journal. pntd.0006015.

7. Rodríguez G, Arenas C, Ovalle C, Hernández C. “Las Leishmaniasis: atlas y texto" Hospital Universitario Centro Dermatológico Federico Lleras Acosta,. Bogotá, Colombia En: Colombia 2016. 1st edition: Editorial Panamericana ISBN: 978-958-59331-0-1 p. 91-106.

8. Saldanha MG, QueirozA, Machado PRL, de Carvalho LP, Scott P, de Carvalho EM, et al. Characterization of the histopathologic features in patients in the early and late phases of cutaneous leishmaniasis. Am J Trop Med Hyg. 2017; 96(3): 645-652.

9. Achtman JC, Ellis DL, Saylors B, Boh EE. Cutaneous leishmaniasis caused by Leishmania (Viannia) 
panamensis in 2 travelers. JAAD Case Rep. 2016; 2(2): 95-97. doi: 10.1016/j.jdcr.2015.11.018.

10. Craig AG, Grau GE, Janse C, Kazura JW, Milner D, Barnwell JW, et al. participants of the Hinxton Retreat meeting on animal models for research on severe malaria. The role of animal models for research on severe malaria. PLoS Pathog. 2012; 8(2): e1002401. doi: 10.1371/journal.ppat.1002401.

11. Loeuillet C, Bañuls AL, Hide M. Study of Leishmania pathogenesis in mice: experimental considerations. Parasit Vectors. 2016; 9: 144. doi: 10.1186/s13071-016-1413-9.

12. Castilho TM, Goldsmith-Pestana K, Lozano C, Valderrama L, Saravia NG, McMahon-Pratt D. Murine model of chronic L. (Viannia) panamensis infection: role of IL-13 in disease. Eur J Immunol. 2010; 40(10): 2816-2829. doi: 10.1002/ eji.201040384.

13. Rojas JI, Tani E, Orn A, Sánchez C, Goto H. Leishmania (Viannia) panamensis-induced cutaneous leishmaniasis in Balb/c mice: pathology. Int J Exp Pathol. 1993; 74(5): 481-491.

14. Travi BL, Osorio Y, Saravia NG. The inflammatory response promotes cutaneous metastasis in hamsters infected with Leishmania (Viannia) panamensis. J Parasitol. 1996; 82(3): 454-457.

15. Pereira CG, Silva AL, de Castilhos P, Mastrantonio EC, Souza RA, Romão RP, et al. Different isolates from Leishmania braziliensis complex induce distinct histopathological features in a murine model of infection. Vet Parasitol. 2009; 165(3-4): 231-240. doi: 10.1016/j.vetpar.2009.07.019.

16. de Moura TR, Novais FO, Oliveira F, Clarêncio J, Noronha A, Barral A, et al. Toward a novel experimental model of infection to study American cutaneous leishmaniasis caused by Leishmania braziliensis. Infect Immun. 2005; 73(9): 5827-5834.

17. Donnelly KB, Lima HC, Titus RG. Histologic characterization of experimental cutaneous leishmaniasis in mice infected with Leishmania braziliensis in the presence or absence of sand fly vector salivary gland lysate. J Parasitol. 1998; 84(1): 97-103.

18. Escobar P, Matu S, Marques C, Croft SL. Sensitivities of Leishmania species to hexadecylphosphocholine (miltefosine), ET-18-OCH(3) (edelfosine) and amphotericin B. Acta Trop. 2002; 81(2): 151-157.

19. Jha TK, Sundar S, Thakur CP, Bachmann P, Karbwang J, Fischer C, et al. Miltefosine, an oral agent, for the treatment of Indian visceral leishmaniasis. N Engl J Med. 1999; 341(24): 1795-1800.
20. Soto J, Arana BA, Toledo J, Rizzo N, Vega JC, Diaz A, et al. Miltefosine for new world cutaneous leishmaniasis. Clin Infect Dis. 2004; 38(9): 1266-1272.

21. Van Bocxlaer K, Yardley V, Murdan S, Croft SL. Topical formulations of miltefosine for cutaneous leishmaniasis in a BALB/c mouse model. J Pharm Pharmacol. 2016; 68(7): 862-872. 